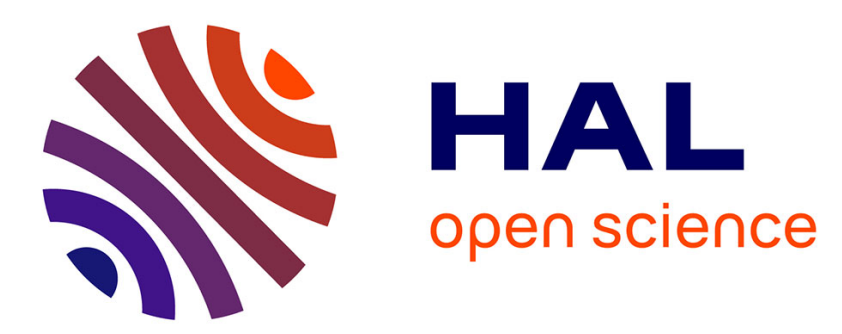

\title{
Apogée et déclin d'un géosystème sylvo-pastoral (Montagne de León et de Palencia, Espagne du nord ouest)
}

Georges Bertrand

\section{- To cite this version:}

Georges Bertrand. Apogée et déclin d'un géosystème sylvo-pastoral (Montagne de León et de Palencia, Espagne du nord ouest). Revue Géographique des Pyrénées et du Sud-Ouest, 1984, Forêts, 55 (2), pp.239-248. 10.3406/rgpso.1984.2979 . hal-02611131

\section{HAL Id: hal-02611131 https://hal.science/hal-02611131}

Submitted on 18 May 2020

HAL is a multi-disciplinary open access archive for the deposit and dissemination of scientific research documents, whether they are published or not. The documents may come from teaching and research institutions in France or abroad, or from public or private research centers.
L'archive ouverte pluridisciplinaire HAL, est destinée au dépôt et à la diffusion de documents scientifiques de niveau recherche, publiés ou non, émanant des établissements d'enseignement et de recherche français ou étrangers, des laboratoires publics ou privés. 


\section{Apogée et déclin d'un géosystème sylvo-pastoral (Montagne de León et de Palencia, Espagne du nord-ouest)}

\section{Georges Bertrand}

\section{Citer ce document / Cite this document :}

Bertrand Georges. Apogée et déclin d'un géosystème sylvo-pastoral (Montagne de León et de Palencia, Espagne du nordouest). In: Revue géographique des Pyrénées et du Sud-Ouest, tome 55, fascicule 2, 1984. Forêts. pp. 239-248;

doi : https://doi.org/10.3406/rgpso.1984.2979

https://www.persee.fr/doc/rgpso_0035-3221_1984_num_55_2_2979

Fichier pdf généré le 20/03/2019 


\title{
Resumen
}

Apogeo y decadencia de un geosistema silvopastoral (Monte de León y Palencia) Geosistema silvopastoral caracterizado por su gran fragilidad natural y una fuerte presión pastoral antigua (siglos XVII-XIX). Caso de inercia y de histéresis. Repoblación de las zonas deterioradas con el roble « tauzin "(Quercus pyrenaica). Este, de atlántico se ha vuelto montañés, pirófilo y heliófilo.

\section{Résumé}

Géosystème sylvo-pastoral caractérisé par une grande fragilité naturelle et par une forte pression pastorale ancienne (XVIle-XIXe s.). Cas d'inertie et d'hystérésie. Recolonisation des surfaces dégradées par le Chêne Tauzin (Quercus pyrenaica), eu-atlantique devenu montagnard, pyrophile et héliophile.

\begin{abstract}
Apex and Waning of a sylvo-pastoral geosystem (mountains of León and Palencia in north western spain). A sylvo pastoral geosystem characterised by a remarkable natural fragility and an old and strong pastoral pressure (17th - 19th centuries). An example of inertia and hysteresis. Reclaiming of degraded areas with the Black Oak (Quercus pyrenaica), a eu-atlantic species that has become montainous, pyrophilous and heliophilous.
\end{abstract}


REVUE GÉOGRAPHIQUE DES PYRÉNÉES ET DU SUD-OUEST TOME 55, FASC. 2, pp. 239-248, Toulouse, 1984.

\title{
Apogée et déclin d'un géosystème sylvo-pastoral (Montagne de León et de Palencia, Espagne du nord-ouest)
}

\author{
par Georges Bertrand *
}

La silhouette fourchue d'un Hêtre isolé, ruisselant dans le brouillard, la touffe d'un Chêne torturé, rampant sur une terre incendiée, un chemin creux aux rives érodées avec, à son détour, la braña, lande infinie dont les ondulations grises se fondent aux nuages : " instantanés " du paysage cantabrique. Et la présence du bétail, bovins, ovins, chevaux et caprins, leitmotiv du paysage : ses bruits, ses couleurs, ses remugles et ses taons agressifs, ses lentes arabesques sur les versants pentus, sa pression dominatrice sur la pelouse, l'arbuste et l'arbre qu'il foule, écrase, arase et mutile... mais aussi fertilise, régénère et restructure.

Entre la " divisoria » qui sépare les affluents du Duero des torrents cantabriques et les hautes plaines de la Vieille Castille, dans les montagnes des provinces de León et de Palencia, entre 900 et $2600 \mathrm{~m}$ d'altitude, s'étendent des paysages divers modelés par l'une des grandes civilisations pastorales, aujourd'hui déchue, du monde ibérique. Paysages relictes, sous-tendus de rémanences et de rétroactions; paysages figés ou paysages réactivés au gré des abandons et des reprises. Peut-être une des clés du fonctionnement du géosystème (1).

* Professeur de géographie, LA 366 CNRS, Université de Toulouse-Le Mirail.

1. G. Bertrand, 1974. Essai sur la systématique du paysage. Les montagnes cantabriques centrales (Nord-Ouest de l'Espagne). 2 tomes, $1160 \mathrm{p}$. Univ. Toulouse-Le Mirail. - C. et G. BERTRAND, 1982. La végétation dans les géosystèmes des montagnes cantabriques centrales. Carte en quadrichromie à 1/200000, imprimée mais non diffusée. 


\section{Entre forêt et lande}

\section{Etagement forestier rompu et forêts confinées.}

La Hêtraie montagnarde acidophile du versant atlantique représente, entre 600 et $1200-1400 \mathrm{~m}$ d'altitude, la seule ceinture forestière à peu près continue. Ailleurs, la forêt est compartimentée en massifs et bosquets qui couvrent à peine un tiers des versants au-dessous de $1600 \mathrm{~m}$ d'altitude. Ici, peu de forêts " pleines " à strates arborées continues et à sous-bois bien individualisés, mais une mosaïque de taillis mal venus, de buissons évolués en halliers, de formations arborées ouvertes, de forêts clairiérées et de parcs. Les arbres, courbés, fourchus, aux multiples itérations, n'ont rien du port forestier. Pourtant leur diversité spécifique et variétale est remarquable : le Hêtre (Fagus silvatica) riche de phénotypes et certainement d'écotypes; les Chênes caducifoliés (Quercus peduncalata, $Q$. sessiliflora, $Q$. pyrenaica) très hybridés; les Chênes sempervirents (Quercus ilex, Q. lusitanica) aux écotypes indéterminés, les Pins (Pinus sylvestris, $P$. insignis) et l'Eucalyptus (Eucalyptus globulus) de reboisement, enfin le Génévrier thurifère (Juniperus thurifera).

\section{Forêts contraintes.}

a. Contraintes mésologiques. L'environnement naturel n'est pas partout favorable à l'arbre et encore moins à la forêt.

Les bioclimats d'altitude, humides, nébuleux, venteux et neigeux, bloquent la limite supérieure de la forêt entre 1200 et $1400 \mathrm{~m}$ sur le versant atlantique, entre 1400 et $1600 \mathrm{~m}$ sur le versant castillan.

La peña, paroi rocheuse, avec son cortège d'éboulements et d'éboulis, ses couloirs d'avalanche remaniés par les torrents, qui s'étend des hautes crêtes jusqu'au fond des gorges. La forêt y est remplacée par des formations arborées ou arbustives.

La prédominance de deux types de substrats peu favorables à la forêt : d'une part, les calcaires massifs dolomitiques très karstifiés; d'autre part, des quartzites et des conglomérats à ciment hématiteux. Les peuplements forestiers ne peuvent atteindre leur masse critique que sur les schistes.

b. Contraintes chorologiques. Malgré sa diversité, le stock floristique régional n'a pas la capacité d'exploiter la totalité des potentialités bioclimatiques et édaphiques.

Citons, pour mémoire, la situation relictuelle du Génévrier Thurifère qui se maintient sur les basses soulanes calcaires. L'absence, mal élucidée, des grands résineux montagnards (Sapin, Pin sylvestre) ou subalpin (Pin à crochets) et la rareté des Bouleaux réduisent l'aire potentielle de la forêt et privent la masse et les marges forestières d'espèces pionnières. 
Le Hêtre, qui constitue la plus grande partie de la biomasse forestière du versant nord, se replie d'abord sur les ombrées des massifs calcaires méridionaux (où la régénération de souche se fait mal). Il se localise ensuite dans des stations relictuelles sur les chaînons proches de la Castille. La Hêtraie cantabrique, la plus méridionale d'Europe occidentale si on excepte la forêt relicte de la Sierra de Gredos, se localise à la "limite maximale ", voire dans la "zone contestée " de l'aire du Hêtre (2). Ces Hêtres n'ont plus aucun pouvoir de dissémination et ils viennent se coincer entre les Chênes verts, les Thurifères et les Sabines (Juniperus sabina).

Les Chênes caducifoliés ne jouent pas un grand rôle forestier à l'exception du Tauzin qui, sous de multiples formes hybridées, est l'arbre des taillis, des forêts ouvertes et des landes arborées. Considéré comme un eu-atlantique strict le Tauzin occupe ici une aire singulière, "ibéro-atlantique montagnarde " (3).

\section{Le * monte bajo ", phénomène diagonal.}

Une mosaïque de formations arbustives, sous-arbustives et herbacée, couvre plus de $60 \%$ de la surface totale des montagnes et assure une certaine continuité physionomique du littoral asturien au piémont castillan : maquis-lande de la Marina, garrigues de Basse Liébana, landes à Fougères et Sarothamne de la moyenne montagne humide, lande-pelouse des puertos (pâturages alpin et subalpin), garides des soulanes calcaires, matorrales des plateaux castillans. Toutefois, l'essentiel du monte bajo est constitué par la braña, lande acidophile dominée par de très nombreuses Ericacées (Erica arborea, E. aragonensis, E. multiflora, E. lusitanica, E. cinerea, etc.), la Daboécie (D. cantabrica), la Bruyère (Calluna vulgaris), les Genêts (Cytisus cantabricus, C. horrida, C. trichomanes), etc. La monotonie physionomique des landes et la sociabilité élevée de leurs espèces ne doivent pas masquer la richesse floristique régionale qui, ajoutée à leur extension territoriale, conduit à s'interroger sur les origines du monte bajo et plus particulièrement de la braña aussi bien dans ses rapports avec les forêts qu'avec les systèmes pastoraux. L'hypothèse d'une formation au moins en partie "climacique " n'est pas à exclure.

\section{La maîtrise pastorale de l'espace}

L'exploitation pastorale a dû commencer dès le Néolithique ; mais les témoignages archéologiques et palynologiques sont ténus et loca-

2. M. BECKER, 1981, in Le Hêtre (sous la direction de $\mathbf{E}$. Teissier du Cros). INRA, Paris, 613 p.

3. P. DUPONT, 1962. La Flore atlantique européenne. Introduction d̀ l'étude du secteur ibéro-atlantique, Toulouse, $414 \mathrm{p}$. 
lisés. Seules, les dernières étapes, celles des $\mathrm{XVIII}^{\bullet}$ et $\mathrm{XIX}^{\bullet}$ siècles qui paraissent, peut-être à tort, capitales, permettent de confronter les études d'archives et les analyses de terrain (4).

\section{La Mesta : l'organisation d'un espace de transhumance.}

A l'exception des maigres champs et des prairies de fauche, d'appropriation individuelle ou régulièrement redistribués, les montagnes sont la propriété des communautés rurales (municipios, concejos). En fait, misère oblige, les montagnes du versant sud cantabrique ont été louées aux grands propriétaires de merinas (moutons à laine) des Castilles, d'Extramadura ou d'Andalousie. Par choix économique royal, la montagne et ses habitants ont été livrés à l'oligarchie pastorale madrilène qui a usé et mésusé de son "droit de location " en introduisant chaque année des centaines de milliers d'ovins sans prendre soin des terrains de pacage et de la forêt. Cette grande transhumance connaît son apogée aux XVII-XVIII siècles avec la mise en place de la Mesta, véritable entreprise de colonisation intérieure, déstabilisatrice des sociétés locales, déprédatrice de ressources naturelles, destructrice de paysages.

Au milieu du XIX ${ }^{e}$ siècle, après la décadence et l'abolition de la Mesta, la transhumance cède la première place à un élevage local, surtout bovin, fondé sur l'estivage et le libre parcours des landes et des forêts. L'accélération de l'exode rural à partir des années 1960 fait tomber la pression pastorale et assure une certaine rémission aux pâturages et aux forêts. Toutefois, le bétail (bovins, ovins, chevaux, chèvres) continue à parcourir la plupart des compartiments du paysage (fig. 1).

\section{L'exploitation forestière sans l'am6́nagement.}

La forêt n'est pas une simple annexe de l'espace pastoral. Les taillis fournissent du bois de feu et les arbres fourchus, aux branches et aux racines courbes étaient destinés à un actif charronnage (instruments aratoires, jougs, chars à bœufs). Les communautés rurales ont toujours pris des mesures de protection, mais apparemment sans grands résultats. Les services forestiers de l'Etat ne se sont pas désintéressés d'une des rares régions d'Espagne pouvant produire du bois de feuillus d'une certaine qualité. Mais il a fallu attendre les années 1950, après les dévastations de la guerre civile, pour que s'élaborent les premiers inventaires forestiers et que soient proposés quelques plans d'aménagement des montes de utilidad pública ainsi que quelques mesures de protection des peuplements les plus menacés (Junipéraies). En fait, l'aménagement forestier débute dans les années 1960-1970 avec la grande politique d'enrési-

4. Enquête historique non publiée réalisée par J.-P. Amalric (Univ. ToulouseLe Mirail). 
nement des brañas par le Patrimonio forestal del Estado, sans souci des communautés rurales, de l'équilibre des milieux montagnards et du devenir des plantations.

\section{Un néo-géosystème sylvo-pastoral}

Les paysages des montagnes de León et de Palencia ressemblent à ceux des façades sèches des montagnes méditerranéennes d'Europe. Leur situation sur la marge océanique humide du domaine méditerranéen permet de mettre en évidence certains de leurs fonctionnements, ou plus exactement certains de leurs dysfonctionnements. L'impression de "versants secs sous climats humides " (5) n'est qu'un raccourci pour exprimer que la combinaison géographique actuelle fonctionne mal et que ses mécanismes ne sont pas encore élucidés : ruptures d'étagement végétal, éclatement de l'espace boisé, extension de landes sans rapport floristique avec les forêts voisines, remontées sur les versants de plantes xérophiles et héliophiles, traces multiples d'érosion...

\section{L'organisation de l'espace sylvo-pastoral.}

a. Les axes pastoraux: cañadas, veredas y cordeles. L'omniprésence pluriséculaire des merinas transhumantes est gravée sur les versants, jusqu'à la roche dure. Sur la " cañada real leonese", grand axe de transhumance de l'Espagne du Nord-Ouest, viennent se greffer des cañadas secondaires qui remontent les grandes vallées (Porma, Cea, Carrión, Pisuerga) pour conduire les merinas jusqu'aux puertos pirenaicos ou puertos de las merinas, pâturages supraforestiers d'excellente qualité qui sont la destination estivale des troupeaux. En fait, les merinas et les troupeaux des villages étaient obligés de séjourner parfois longuement, parfois toute l'année, dans la moyenne montagne : surcharge pastorale des puertos, mauvais temps fréquent dans ces montagnes directement exposées aux masses d'air océaniques (enneigements précoces ou tardifs, etc.). De nombreurses veredas se détachent des cañadas, traversent villages et cultures, franchissent des gués et des cols et par des cordeles irradient toute une partie de la moyenne montagne, et plus particulièrement les soulanes.

b. Le feu-outil (6). La Mesta est abolie, les stratégies économiques ont changé, mais la pratique pastorale est restée immuable. Le feu, suivi du piétinement et de la fumure du bétail, a été et reste l'irremplaçable outil pour obtenir du pâturage : feu courant contrôlé dans les landes et les pelouses arbustives; petits feux de nettoyage des sous-bois ; feux chauds des broussailles et des taillis; mais aussi

5. G. Bertrand, 1972. Ecologie d'un espace géographique : les géosystèmes du Valle de Prioro (Espagne du Nord-Ouest). L'Espace géographique, n 2, p. 113-128.

6. Etude en cours de J.-P. Métailié. Voir, par aillcurs, du même, Le feu pastoral dans les Pyrénées Centrales (Barousse, Oueil, Larboust), 1981. Toulouse, 293 p. 


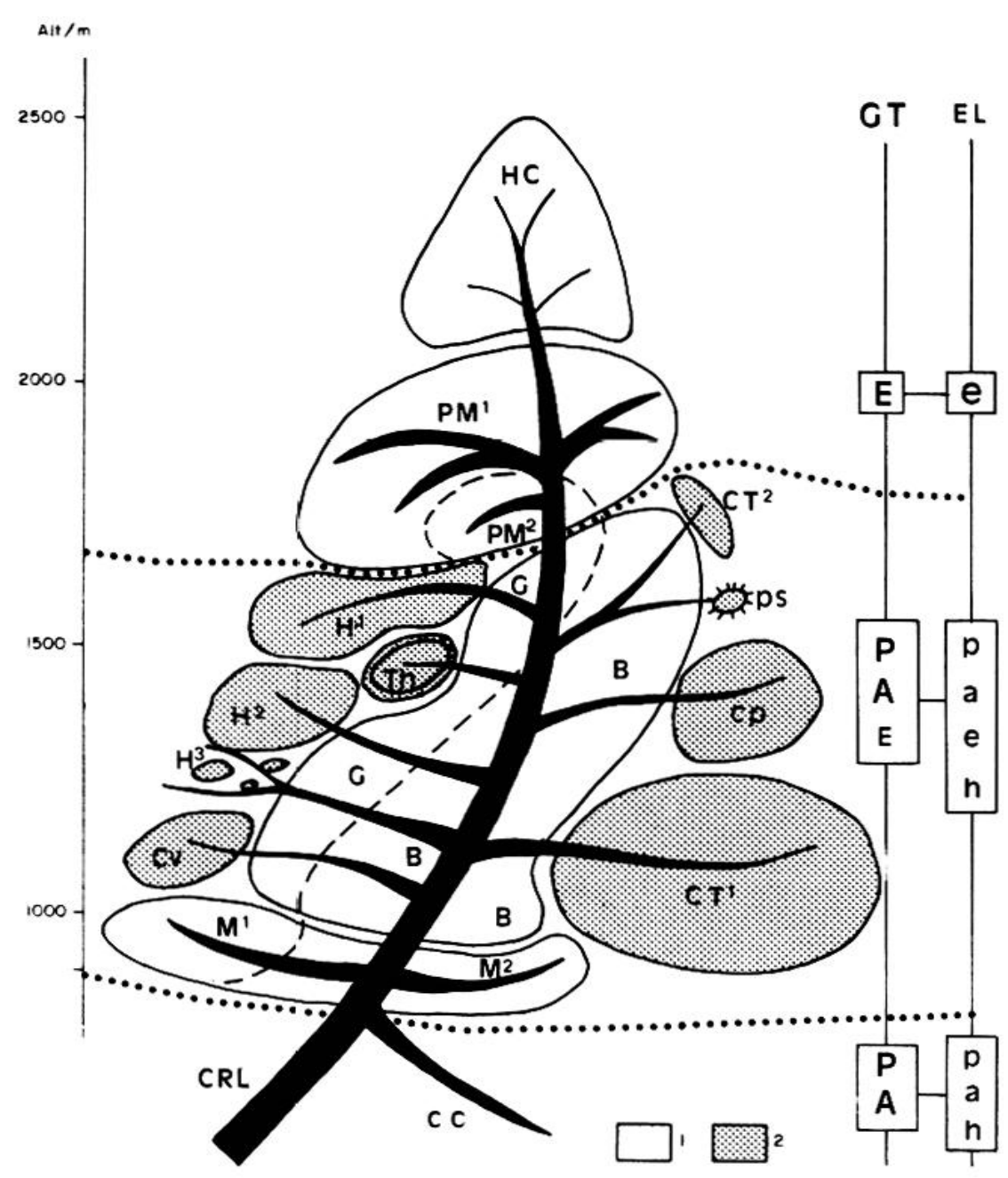

FIG. 1

Répartition de la cherge pastorale par milieux ot par saisons (tendance générale depuis le $\mathrm{XVII}^{\circ}$ siècle au moins).

1. Formations basses et ouvertes pastoralisées. - 2. Bois et forêts.

La grande flèche représente schématiquement le système des "cañadas ", "veredas » et " cordeles " (CRL : Cañada Real Leonesa, CC: Cañada Cerverena).

Milieux: HC: Hautes crêtes - PM1 : "Puertos de las merinas " (pâturages supraforestiers), sur substrat siliceux. - $\mathrm{PM}^{2}:$ Idem sur substrat calcaire. - $\mathrm{H}^{1}$ : Hêtraie montagnarde acidophile humide. - $\mathbf{H}^{2}$ : Hêtraie montagnarde calcicole mésophile. - $\mathrm{H}^{3}$ : Hêtraie montagnarde calcicole rélictuelle. - ps : Plantation de Pin sylvestre (XVIII siècle ?). - cp : Chênaie montagnarde à Chêne pédonculé - Chêne sessile. - CT' 1 : Chênaie montagnarde à Chêne tauzin. - B: Braña » (landes acidophiles). - G : Garides (frutex sur substrat calcaire). M1 : Matorral à Chêne vert, sur substrat calcaire. - M2 : Idem sur substrat siliceux.

Saisons de parcours: pour la grande transhumance: $\mathrm{P}, \mathrm{E}, \mathrm{A}, \mathrm{H}$; pour les élevages locaux: $p, e, a, h$ (printemps, été, automne, hiver).

brûlis culturaux itinérants qui se sont perpétués localement jusque vers les années 1950; enfin, quelques incendies dans les jeunes plantations de résineux. 


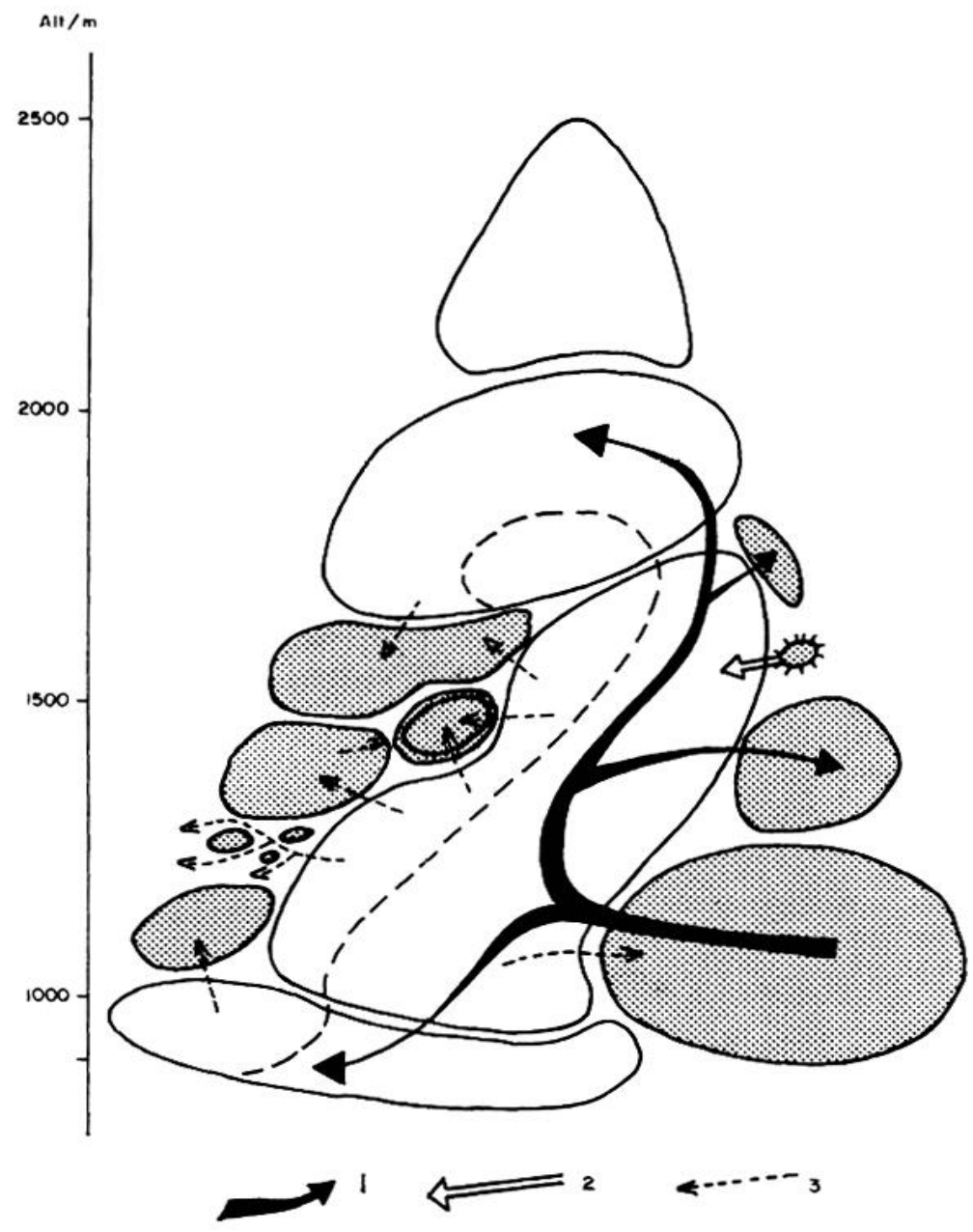

Fig. 2

Fonctionnement spatial du géosystème agro-pastoral (1960-1980)

1. Progression du Chêne Tauzin en touffes, buissons et taillis. - 2. Progression du Pin sylvestre. - 3. Dégradation pastorale en cours des bois et des forêts.

c. Le Chêne Tauzin pastoral et pyrophile. Le Tauzin, ou du moins le Chêne à caractères dominants et apparents de Tauzin, est l'arbre, sinon l'arbuste et le buisson, le plus représentatif de l'espace sylvopastoral. Non seulement il constitue quelques taillis monospécifiques, certains d'assez belle venue (hauteur: $15 \mathrm{~m}$, diamètre : $0,25 \mathrm{~m}$, âge \pm 50 ans), mais encore il s'insinue isolément ou par touffes dans les landes et les pacages ouverts par le feu, en concurrence directe avec les Ericacées et la Callune. Il colonise tout le versant sud depuis les matorrales à Chêne vert jusqu'à la limite de la lande alpine à $2250 \mathrm{~m}$ d'altitude où il progresse sous forme de coussinets. La Hêtraie est repliée sur les ombrées calcaires. Avec J.-P. Métailié, nous proposons, pour en rendre compte, l'hypothèse d'une espèce secondairement pyrophile et héliophile, susceptible de coloniser 
rapidement par reproduction végétative les substrats acides incendiés et surpâturés. Enfin le Tauzin est un arbre fourrager. Le bétail mange les feuilles et les tiges tendres et la plupart des vieux Tauzins présentent le port en chandelier des arbres émondés (fig. 2).

\section{Géofaciès sylvo-pastoraux.}

Ils se répartissent de façon discontinue le long des axes pastoraux. Nous ne citerons ici, à titre d'exemple que quelques-unes des unités les plus significatives:

Rañas du piémont castillan, à $900 \mathrm{~m}$ (vers Boñar) : Matorral pyrophile et surpâturé (7) composé ainsi :

4. Quercus ilex $1-3$. Q. pyrenaica. $2-2$. Cistus ladaniferus, Calluna vulgaris, Lavandula pedunculata, Asphodelus albus. 2-3 1. Graminées. + (saison sèche).

Versant schisteux du bassin de Cervera, vers $550 \mathrm{~m}$ : reposoir à Tauzins nanifiés $(1,50 \mathrm{~m}-2 \mathrm{~m})$ et brûlés par l'excès d'urine et de fèces, tiges et troncs tortueux, feuillage en toiture.

Taillis pâturé de Tauzin-Sessile sur sol brun acide évoluant en jeune gaulis, à Cervera, vers $1100 \mathrm{~m}$. Sous-bois herbacé (Agrostis, Festuca) entretenu par la technique de "petits feux".

Replats de rañas du bassin de Riaño, vers $1200 \mathrm{~m}$ : brûlis pastoral d'un an, fort recrû de Tauzin sur tiges incomplètement brûlées ou directement sur souche, rares repousses de Callune et d'Helianthème.

Versant sur schistes, à Riaño, vers $1250 \mathrm{~m}$ : Lande monospécifique à Erica aragonensis sur pavage; vieux Tauzins (250 - 350 ans ?) isolés, cimes mortes ( 5 à $12 \mathrm{~m}$ de haut, 1 à $3 \mathrm{~m}$ de diamètre).

Soulane calcaire, à Besande, vers $1300 \mathrm{~m}$ : Hêtraie détruite et sols bruns décapés; Garide couverte à Ononis spinosa, Thymus mastichina, quelques Hêtres en boule ou en buisson.

Versant d'ombrée du Valdeburón sur schistes altérés et moraines à $1450 \mathrm{~m}$ : lisière de Hêtraie ainsi constituée :

3-4: Fagus silvatica et Ilex aquifolium abroutis en quenouilles. 2. - 2-3: Cytisus sarothamnus, Pteridium aquilinum. 2. -1 : Festuca. 4.

Cañada, Valdetuejar, vers $1150 \mathrm{~m}$ : ravinements métriques dans les schistes: Marrubium, Santolina pectinata.

\section{Conclusion : un géosystème sylvo-pastoral en état d'hystérésis}

Cet ensemble sylvo-pastoral physionomiquement hétérogène recoupe en diagonale les géosystèmes plus ou moins étagés du versant sud

7. Le premier chiffre indique la strate (de 0 à 7 ), le second l'abondancedominance par strate (de 0 à 5 ). 
cantabrique (sierras méridionales, bassins intramontagnards, hauts massifs, etc.). Il emprunte à chacun de ces géosystèmes des géofaciès qui se caractérisent chacun par des topoclimats, des substrats, des sols et des cortèges floristiques différents. Peut-on dans ce cas parler d'un géosystème?

\section{L'état et le fonctionnement d'un géosystème.}

La fragilité des milieux naturels et l'absence de nombreuses essences forestières expliquent, pour une large part, l'état de dégradation, voire de déséquilibre. Mais c'est le système pastoral, plus exactement l'état de surpâturage, qui est à l'origine de ces nouveaux paysages marqués par le bétail et par le feu. Avant que d'être physionomique l'unité est fonctionnelle.

\section{L'histoire du géosystème : inertie et hystérésis.}

La charge pastorale actuelle est trop faible pour expliquer l'état du géosystème et son fonctionnement. Ce géosystème est apparu il y a à peine quelques siècles sous les effets de la transhumance et il continue à fonctionner sur lui-même. L'évolution des vingt dernières années, avec la diminution de la charge pastorale, est caractérisée par deux phénomènes non contradictoires.

D'abord, une inertie assez générale. Les géofaciès sont encore sous "tension pastorale ". Il n'y a pas de vague d'enfrichement et encore moins de front pionnier forestier de type pyrénéen (8). Cette inertie écologique revêt ici une telle importance que l'on doit se poser le problème de l'irréversibilité de certains processus physiques ou biologiques engagés au moment du maximum pastoral (décapages de manteaux d'altération et de sols hérités, disparition d'essence forestière et d'espèces herbacées).

Ensuite l'hystérésis de certains géofaciès qui ont franchi un seuil d'instabilité (ouverture des strates végétales inférieures avec mobilisation des dépôts meubles, etc.). Sans cause apparente certains processus s'accélèrent et font boule de neige: décapage des sols bruns sous la Hêtraie, ramonage des versants calcaires par des avalanches qui emportent les derniers bouquets de Hêtres, etc.

Ce "néo-géosystème " fonctionne aujourd'hui autant par inertie que par rémanence et il est entré dans la première phase de son déclin. Mais les processus qui le commandent sont pour la plupart irréversibles, au moins à l'échchelle séculaire et sa récupération par les forestiers paraît des plus difficiles. Fortement destructeur, il n'en est pas moins créateur de ressources et de paysages. Le Tauzin n'est-

8. C. Suffert-Carcenac, 1978. Les grandes unités éco-agrologiques des Pyrénées françaises en 1970-1978. Esquisse cartographique au 1/25000, RGPSO, 49, 4, pp. $443-454$. 
il pas ici un écotype lié au feu et au piétinement du bétail ? Il représente moins un ancien témoin de la forêt qu'un colon capable de consolider les forêts existantes, de gagner sur les landes et les brûlis en constituant une certaine ressource fourragère. Il faudrait mieux le connaître pour pouvoir le gérer dans la forêt et hors la forêt.

RESUME. - Géosystème sylvo-pastoral caractérisé par une grande fragilité naturelle et par une forte pression pastorale ancienne $\left(\mathrm{XVII}^{\mathrm{e}}-\mathrm{XIX}^{\mathrm{e}} \mathrm{s}\right.$.). Cas d'inertie et d'hystérésie. Recolonisation des surfaces dégradées par le Chêne Tauzin (Quercus pyrenaica), eu-atlantique devenu montagnard, pyrophile et héliophile.

Summary, - ApeX and Waning of a sylvo-pastoral geosystem (MOUNTains of León AND PALENCIA IN NORTH WESTERN SPAIN). A sylvo pastoral geosystem characterised by a remarkable natural fragility and an old and strong pastoral pressure (17th - 19th centuries). An example of inertia and hysteresis. Reclaiming of degraded areas with the Black Oak (Quercus pyrenaica), a eu-atlantic species that has become montainous, pyrophilous and heliophilous.

Resumen. - Apogeo y decadencia de un geosistema silvopastoral (MoNTe de Lén Y PALENCIA) Geosistema silvopastoral caracterizado por su gran fragilidad natural y una fuerte presión pastoral antigua (siglos XVII-XIX). Caso de inercia y de histéresis. Repoblación de las zonas deterioradas con el roble " tauzin " (Quercus pyrenaica). Este, de atlántico se ha vuelto montañés, pirófilo y heliófilo.

Mots-Clés. - EsPagne, Chaîne cantabrique, GÉosysteme, montagne, forêt, pâturage, systême sylvopastoral, dégradation, feu, époque moderne et contemporaine. 